\title{
The use of tablets and its influence in the motivation of 12-13-year old students: gender differences
}

\author{
Miriam Mendez, David Mendez, Juana Anguita. \\ Universidad Alfonso X el Sabio
}

\begin{abstract}
:
The Self-Determination Theory (Deci and Ryan, 1985) is based on intrinsic motivation. It states that when students who are not motivated start using tablets in the classroom, they are more motivated than those using textbooks (Mendez, Mendez and Anguita, 2018). Hence the use of technology could be a solution. But do these devices cause the same effect on the motivation of girls and boys? On the basis of this theory, the test based on Self-Determination Theory was given to 12 and 13-year old students from two different schools. The students used tablets in the Science class. The sample consists of 131 students, 58 girls and 73 boys. The test measured three dimensions of intrinsic motivation: interest and satisfaction with the tasks the students had to perform, how they perceive their competence to use the tablet and the value of the tasks they have to carry out with the tablet. Boys are more motivated than girls in the first two dimensions, whereas girls are more motivated than boys in the third dimension.
\end{abstract}

Tablet, motivation, gender differences.

Keywords: motivation, tablet, gender differences. 